\title{
CUSTOMISED LIGHT SOURCE FOR SINUSCOPE A NOVEL DESIGN
}

\section{Authors}

Arvind Kumar Verma(1), Abhishek Bahadur Singh(2), Angira Katyayan (3)

1 Resident, 2Assistant Professor, Department of Otolaryngology, King George Medical University Lucknow and 3Btech Intern from SRM Institute of Technology, Kattankulathur, Chennai

\begin{abstract}
:
Sinuscopes are durable instruments that deliver excellent visualization needed for diagnostic, therapeutic and surgical sinus and nasal procedures. It requires a light source like all other endoscopes for illumination of the objects.

In lieu of expensive cost of commercial external light source we have designed a hand held customized low cost light source for sinuscope to be highly cost effective in routine practice particularly at places with poor infrastructure. This customized light source consists of hallow metallic tube fitted with LED bulb connected with battery which description we are sending for its patenting before presenting it for publication
\end{abstract}

\section{Specification:}

Sinuscopes are in regular practice these days especially owing to their subsidised cost. The associated hand-held light source (Figure 1) has replaced the external light source with cable connection particularly in outpatient clinics during routine patient examination. The average cost of the hand-held light source is estimated to be somewhere from 10,000 to 15,000 INR with further increment in cost as the rechargeable battery needs replacement. Our team presents a basic design that costs less than 100 INR and is operated with simple slender torch batteries.

The diagrammatic outline of the design is depicted in (Figure 2). The 3 components (Figure
3) of this devise are (1) a hollow 4 inch rigid metallic tube with diameter adequate to fit snugly over the 'light-source' terminal of sinuscope and also lodge a small bulb, (2) flat LED bulb with soldered wires at positive and negative terminals (the LED bulb is just enough to be negotiated inside the tubular lumen), (3) Slender torch battery that can be strapped to the metallic tube and the wires connected accordingly.

The metallic tube is snugly fitted over the 'lightsource' terminal of sinuscope and the LED bulb is inserted inside the metallic tube just up to a distance that can nearly touch the 'light-source' terminal of sinuscope. The wires are delivered out of the other end and then connected to the battery strapped outside of the tube (by some switching mechanism if possible). The quality of image obtained through this devise is more or less the same as that obtained with the commercially available expensive devise (Figure 4).

The total cost of this customised devise is less than 100 INR as compared to its expensive counterpart and can be managed by ordinary batteries. This is particularly desirable at peripheral centers where power cuts do not allow a proper recharge of the rechargeable battery. With the availability of extremely cheap sinuscopes, their integration with this newly designed light source is likely to improve nasal diagnosis in the community. 


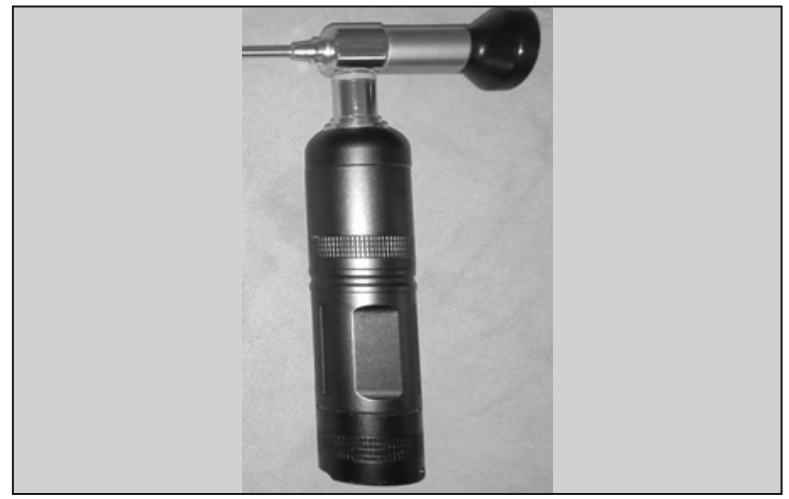

Figure 1: Commercial hand-held rechargable external light source fitted with the sinuscope along the light-source terminal.

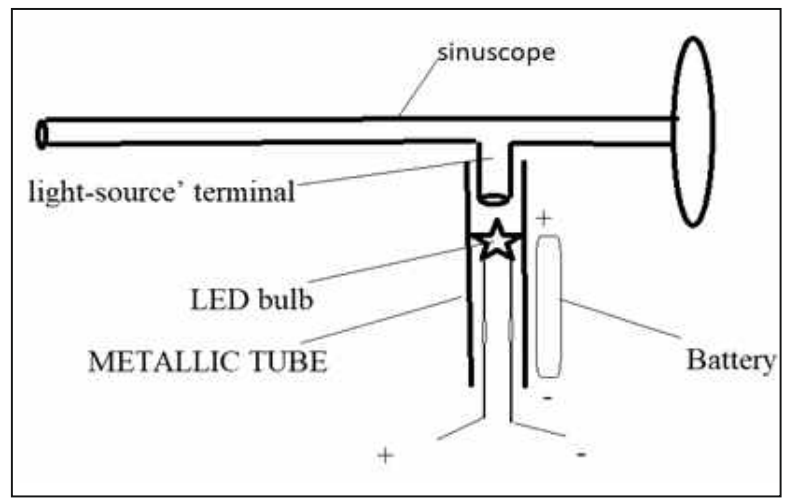

Figure 2: Three parts of the novel design (1) Metallic rigid tube that lodges the (2) LED bulb that is connected with wires to the (3) battery strapped along the metallic tube. Note that the metallic tube snugly fits onto the light-source -terminal of sinuscope.

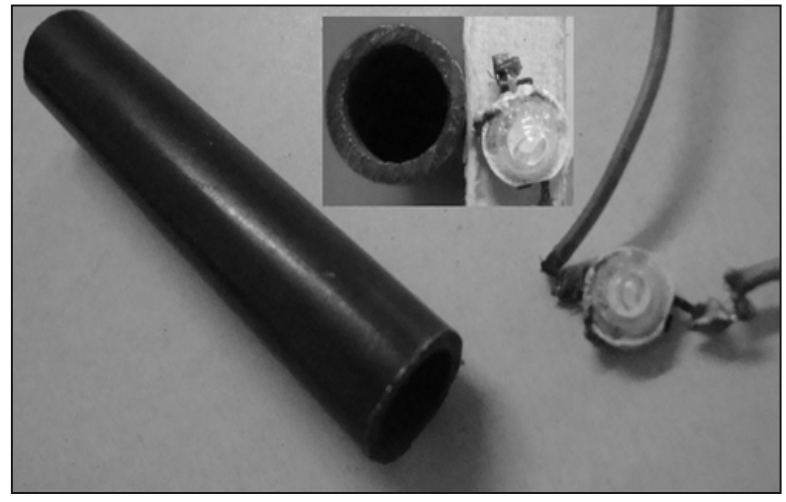

Figure 3: A hollow metallic tube and flat type LED bulb with wire connection. The inset depicts the diameter of the metallic tube that is compatible with the diameter of LED bulb.
Figure 4: Comparison of the quality of image with commercial hand-held rechargeable light source (left) with the image as seen by using the novel design.

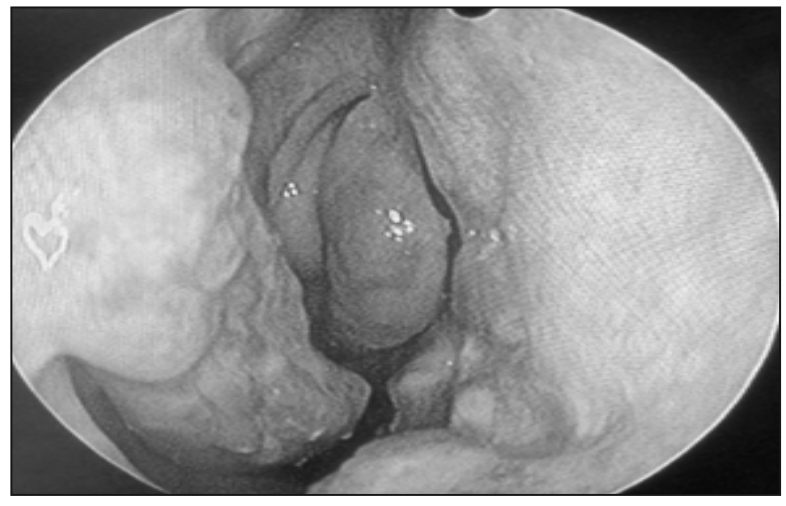

Picture with Novel design light source

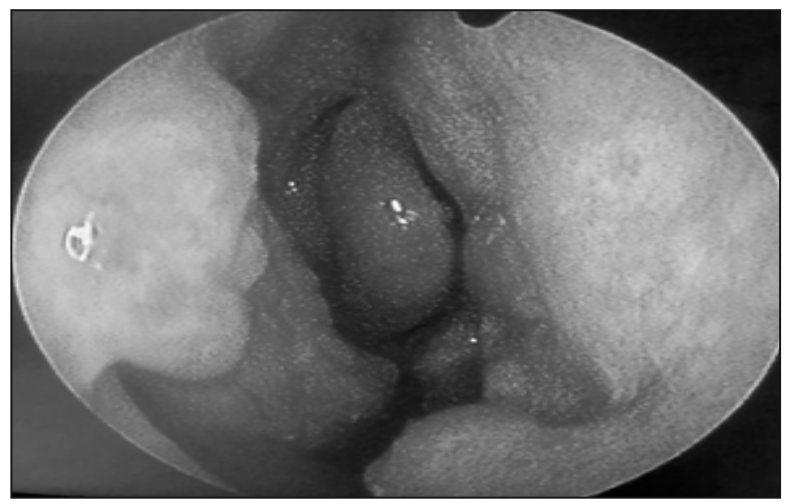

Picture with commercial hand-held rechargeable light source

\section{Conclusion}

In lieu of extremely low cost and easy availability of all parts in such customized light source with time to time refinement would become very promising instrument in near future.

\section{Corresponding author:}

Dr. Abhishek Bahadur Singh, Assistant Professor, Department of Otolaryngology, King George Medical University Lucknow.

An application for patenting this design has been submitted. 